\title{
Outcome of Surgical Management of Sacrococcygeal Pilonidal Sinus Disease with Rotation Flap in 52 Patients-A Retrospective Study
}

\author{
Abbas Mistry ${ }^{1} \quad$ Parvez Shaikh² \\ George Anthony ${ }^{2}$ \\ ${ }^{1}$ Department of Plastic and Reconstructive Surgery, Saifee Hospital, \\ Mumbai, Maharashtra, India \\ ${ }^{2}$ Department of General Surgery, Saifee Hospital, Mumbai, \\ Maharashtra, India \\ ${ }^{3}$ Department of Anesthesiology, Saifee Hospital, Mumbai, \\ Maharashtra, India \\ Indian J Plast Surg 2021;54:163-167.
}

\author{
Samir Bagasrawala ${ }^{2}$ Ankit Chauhan²
}

\begin{abstract}
Address for correspondence Abbas Mistry, MBBS, MS, MCh, Department of Plastic and Reconstructive Surgery, Saifee Hospital, 1001, Rekab Towers, 20 E.S.P Marg, Ghodapdev, Mumbai 400033, Maharashtra, India (e-mail: drabbasmistry@gmail.com).
\end{abstract}

\begin{abstract}
Keywords

- pilonidal sinus

- rotation flap

Background Surgical treatment of sacrococcygeal pilonidal sinus disease (SPSD) consists of radical excision of the entire tract and treatment of the resultant raw area. Here, the authors have reviewed the results of the rotation flap for closure of the SPSD. This study aims to evaluate the outcomes following SPSD excision and rotation flap closure.

Materials and Methods All patients were treated for SPSD with excision and closure using a rotation flap from January 2010 to September 2018. Cases having a follow-up of at least 6 months post surgery were evaluated.

Result A total of 52 patients were included in the study; 42 cases were of primary disease while 10 were of recurrent disease. The patients' follow-up records on the 3rd day, 10th day, 1 month, and 6 months were evaluated.

None of the patients showed any signs of recurrence on follow-ups. One patient developed a hematoma on the third day post surgery which was treated conservatively. One patient developed a seroma in the perianal region on the fifth postoperative day which required aspiration. Both these patients healed well subsequently.

Conclusion Rotation flap is a (simple and reliable) treatment option for closure of postexcision SPSD defect. It not only takes the tension away from suture line, but also pushes the gluteal fat from the sides into the midline, obliterating the deep crevice of the natal cleft which is believed to be one of the important factors in the causation of SPSD, thus minimizing recurrence.
\end{abstract}

\section{Introduction}

Sacrococcygeal pilonidal sinus disease (SPSD) is characteristically a blind epithelial tract generally containing hair.
DOI https://doi.org/ $10.1055 / \mathrm{s}-0041-1729506$ ISSN 0970-0358
It is believed to be caused by excessive hairiness and poor hygiene. ${ }^{1}$ Other factors affecting the incidence are increased sweating associated with sitting and buttock friction, obesity, and local trauma. Increased depth, narrowness of the natal 
cleft, and the friction movements of the buttocks pave the way for loose hair to collect and insert into the skin of the natal cleft. The hair is perceived as a foreign body, and this initiates an inflammatory response that can then lead to a pocket of infection, leading to an abscess or sinus formation.

The prevalence of SPSD is $4 \%{ }^{12}$ As observed by Akinci et $\mathrm{al},{ }^{3}$ SPSD has a predilection for patients having a deeper natal cleft.

Surgical excision of the entire sinus tract is the mainstay treatment option for SPSD. Several methods have been described for the closure of the postexcision SPSD defect which include vy-plasty, z-plasty, and w-plasty. However, no consensus exists on a standard method for closure. In this study, the authors have evaluated their results of performing a rotation flap for the closure of postexcision SPSD defect. Rotation flap has been in used in plastic surgery, ${ }^{4}$ but its use in SPSD was reported only once. ${ }^{5}$ Other publications claiming rotation flap for the treatment of SPSD are in fact transposition flaps by definition. ${ }^{6}$

\section{Materials and Methods}

Fifty-two patients treated for SPSD with excision and closure using rotation flap from January 2010 to September 2018 were included in this study as per the inclusion criteria:

1. Primary cases of SPSD with a postexcisional defect of $4 \mathrm{~cm}$ or more in its transverse diameter (width), irrespective of length.

2. Recurrent cases of SPSD treated by any other method at least 6 months prior.

Once the diagnosis was made, on OPD (out patient department) basis, pus was sent from the sinus opening for bacterial culture and antibiotic sensitivity for selecting the antibiotic during perioperative period. In case of "no growth," amoxycillin and clavulanic acid were our drugs of choice. The patient was admitted in daycare.

\section{Surgical Technique}

Patients were placed in prone jack knife position. Methylene blue mixed with hydrogen peroxide was instilled through the sinus opening to define the extent of the ramifications of the sinus ( - Fig. 1). The area to be excised was carefully marked and the rotation flap was mapped on the skin. As with any rotation flap the length of the arc of rotation was around six times the length of the width of the base of the triangulated defect ( - Fig. 2).

This surgery was done under sedation and local anesthesia. A solution for local infiltration was prepared by mixing $30 \mathrm{~cm}^{3}$ of lignocaine $2 \%$ with $20 \mathrm{~cm}^{3}$ of bupivacaine $0.5 \%$. $1 \mathrm{~cm}^{3}$ of adrenaline was added to this solution ( - Fig. 3). Any remnant of the sinus tract, as visualized by the staining with methylene blue, was also excised. The defect was triangulated.

The rotation flap was designed adjacent to the defect and was rotated about a fixed pivot point to resurface the defect. It is based on random pattern vascularity. Up to $8 \mathrm{~cm}$ defect (length of the base of the triangulated defect) can be closed

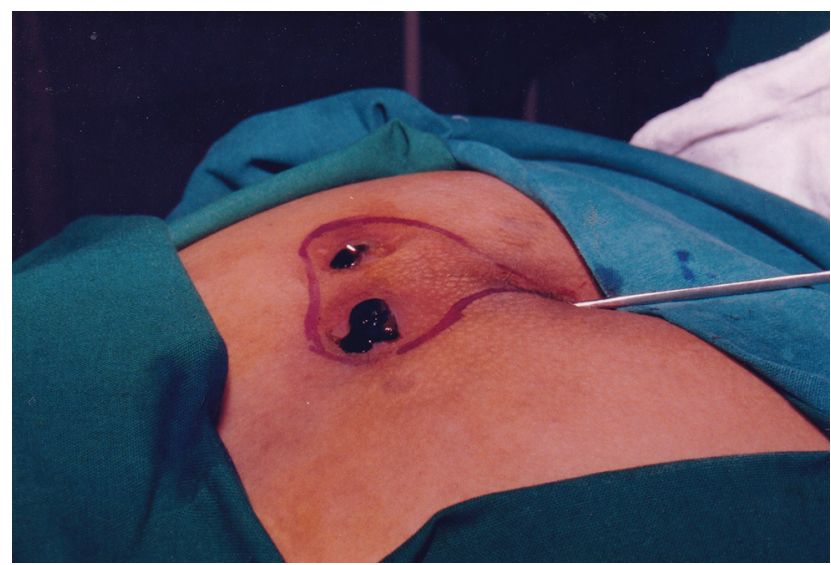

Fig. 1 Sacrococcygeal pilonidal sinus disease (SPSD) with sinus tract marked with methylene blue and hydrogen peroxide.

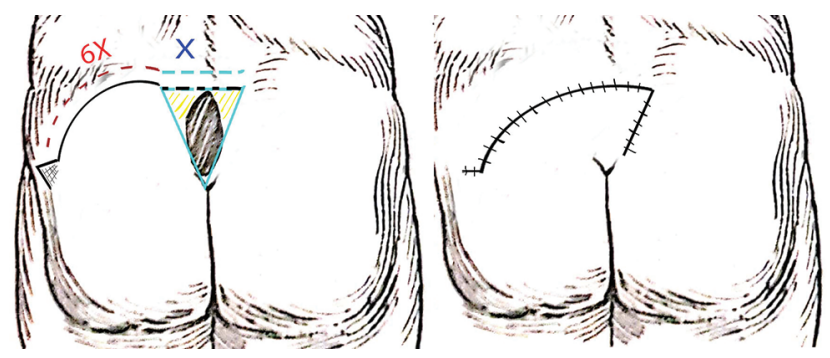

Fig. 2 Flap design; for additional advancement a triangle may be excised at the lateral end of the flap.

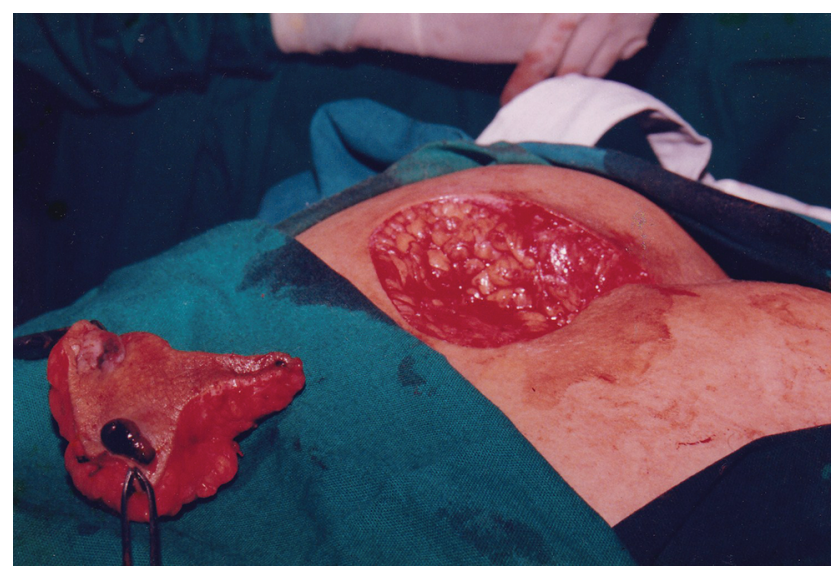

Fig. 3 Post excision: specimen and defect.

primarily. In case if there is tension on suture line, one can do additional advancement by excising a triangle from the lateral aspect of the base of the pedicle. ${ }^{7}$ This has advantage over a back cut which also allows additional advancement but not at the cost of decreasing the width of the pedicle of the flap, thus endangering the vascularity ( - Fig. 2 ).

The flap was rotated to cover the defect ( - Fig. 4) and suturing was done in 2 layers-subcutaneous and subdermal layer with 2-0 polyglyceparone (absorbable) sutures and skin with staples. A closed drainage system was employed. The patient was discharged on the same evening.

These patients were advised 1 week bed rest. 


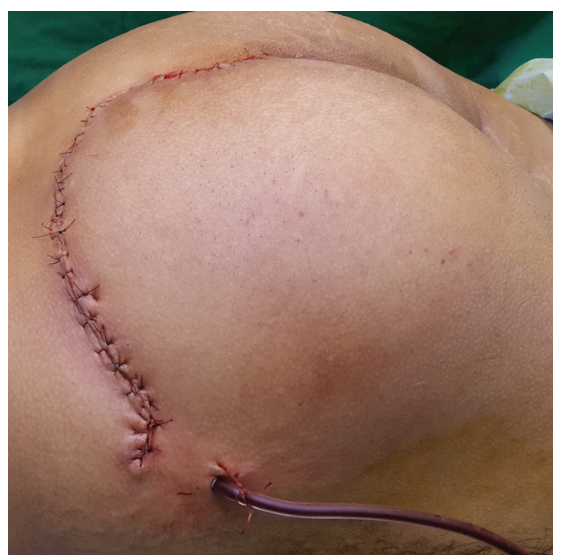

Fig. 4 Rotation flap completed with drainage tube.

They were recalled for follow-up after 48 hours to check for any hematoma and removal of drain. Further follow-up was after 8 days for removal of staples, and subsequently every month for the first 6 months and thereafter every 6 months (-Fig. 5).

Records of the patient follow-ups on the 3rd day, 10th day, 1 month, and 6 month post surgery were evaluated ( - Fig. 5 ).

\section{Results}

A total of 52 patients were included in this study. Their age ranged from 19 years to 36 years (mean $29.4 \pm 5.5$ years). Forty were males and 12 were females. Forty-two cases were of primary disease and 10 were of recurrent disease.

As evaluated intraoperatively, the transverse defect, post excision, ranged from $4 \mathrm{~cm}$ to $10 \mathrm{~cm}$, with the mean $9.4 \pm$ $1.14 \mathrm{~cm}$.

As evaluated on the third day post surgery, 1 patient out of 52 had hematoma formation, which was treated conservatively, while the rest had shown uneventful healing.

As evaluated on the fifth day post surgery, 1 patient developed a seroma in the perianal region that required aspiration. However, subsequent healing was uneventful.

At 1 month post surgery follow-up, all 52 patients had no signs of any recurrence of the disease and a healthy suture line.

At 6 month post surgery follow-up, all 52 patients had no signs of any recurrence.

Laser depilation of the back and trunk was undertaken for all our patients.

\section{Discussion}

The surgical treatment of SPSD should intend toward removing all the sinus tracts as well as the predisposing factors that contribute to the formation of pilonidal sinus. A major predisposing factor is the deep groove of the natal cleft which needs to be obliterated ${ }^{8}$ ( - Figs. 6 and 7 ).

The treatment described in literature varies from conservative to complicated musculocutaneous flaps depending on the presentation of the disease and the competence of the surgeon. Some authors treat patients with abscess and

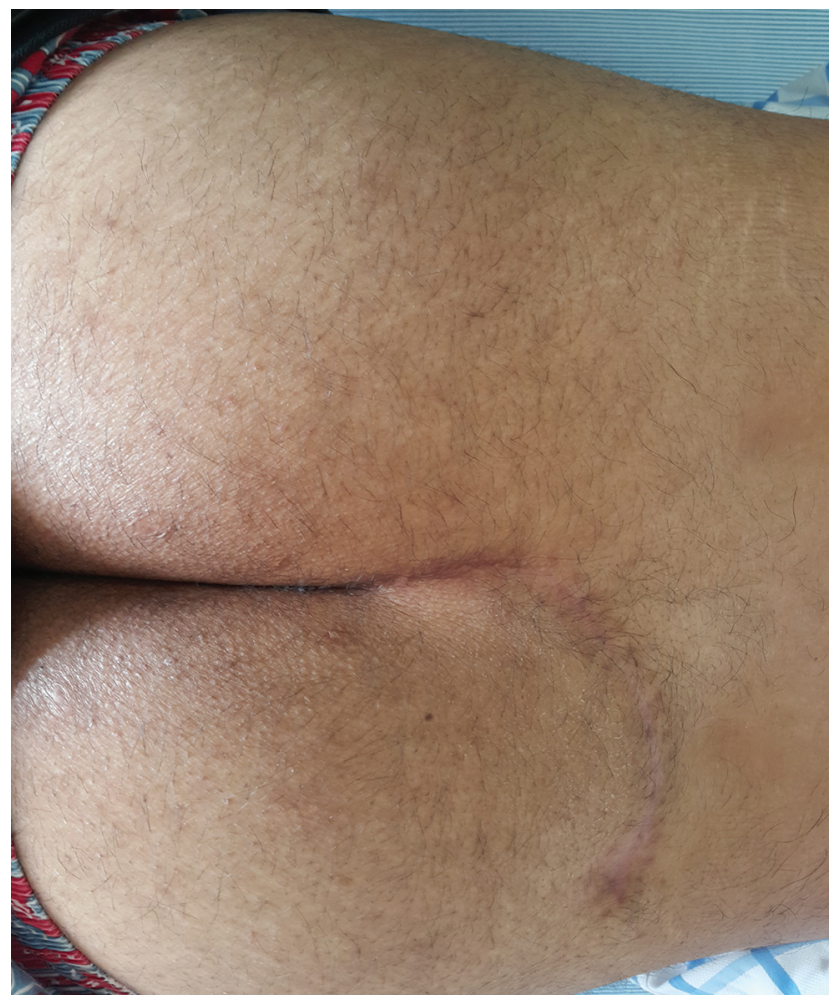

Fig. 5 Long-term results 1.

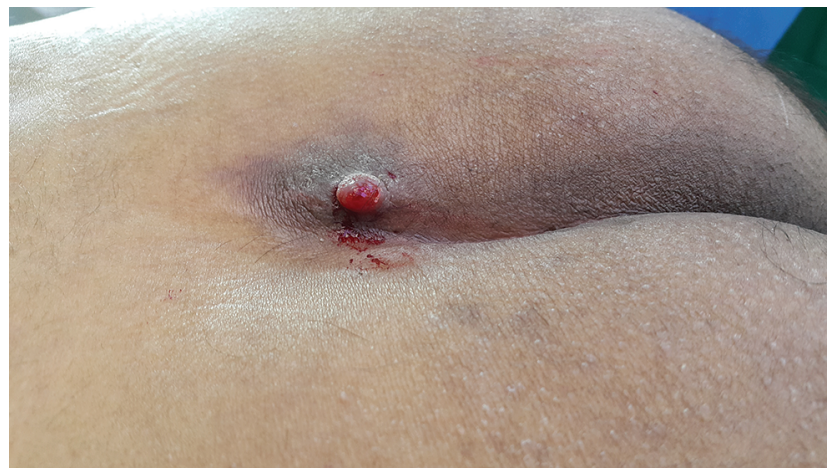

Fig. 6 Deep groove in natal cleft in patients with sacrococcygeal pilonidal sinus disease (SPSD).

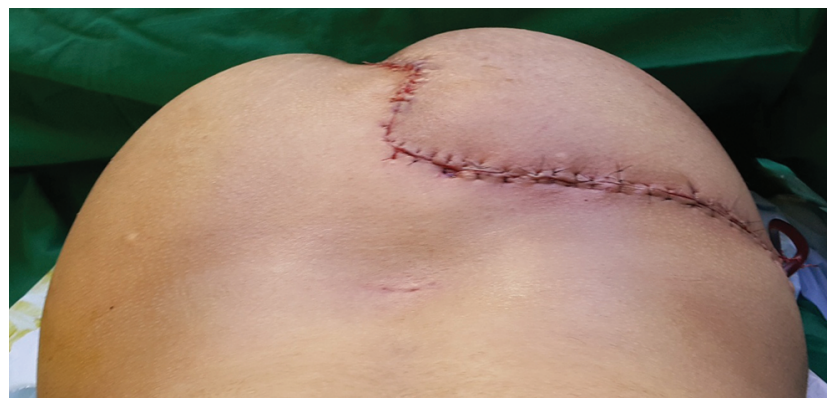

Fig. 7 Groove being totally obliterated after surgery.

active infection in two stages, the definitive repair being the second stage.

In an extensive review from 1945 to 2017 by Johnson et al1 $1^{9}$ it has been suggested that primary closure is better than marsupialization or secondary closure ${ }^{10}$ in terms of recurrence. 
Table 1 Compilation of results of previous studies by other authors to ours for the surgical management of sacrococcygeal pilonidal sinus disease (SPSD)

\begin{tabular}{|c|c|c|c|c|c|c|}
\hline Operation & Author & $\begin{array}{l}\text { Anesthesia } \\
\text { type }\end{array}$ & $\begin{array}{l}\text { Length of hospital } \\
\text { stay (days) }\end{array}$ & $\begin{array}{l}\text { Follow-up } \\
\text { (years) }\end{array}$ & $\begin{array}{l}\text { Morbidity } \\
\text { (\%) }\end{array}$ & $\begin{array}{l}\text { Recurrence } \\
\text { (\%) }\end{array}$ \\
\hline z-Plasty (transposition) & Monroe and MacDormett ${ }^{14}$ & GA & 21 & - & - & 0 \\
\hline z-Plasty (transposition) & Toubanakis $^{15}$ & - & - & $1-10$ & 0 & 0 \\
\hline w-Plasty (transposition) & Roth and Moormen ${ }^{16}$ & GA & 5.7 & - & - & 8 \\
\hline $\begin{array}{l}\text { Asymmetric incision } \\
\text { (off-midline) }\end{array}$ & Karydaikys $s^{17,18}$ & GA & 3 & $2-20$ & 8.5 & $>1$ \\
\hline $\begin{array}{l}\text { Asymmetric incision } \\
\text { (off-midline) }\end{array}$ & Bascom $^{19}$ & - & - & - & $8.5-9$ & $1-4$ \\
\hline V-Y advancement & Khatri ${ }^{21}$ & & 5 & $0.4-4.5$ & & \\
\hline Rhomboid flap & Milito $^{23}$ & GA/SA & 5.3 & 6.2 & 3 & 0 \\
\hline Elliptical rotation Flap & Omer $^{6}$ & - & 1 & - & $0.8-4.1$ & 0 \\
\hline $\begin{array}{l}\text { Rotational advance- } \\
\text { ment flap }\end{array}$ & Mistry & $\begin{array}{l}\text { LA/ } \\
\text { sedation }\end{array}$ & 1 & $0.5-5$ & 3.8 & 0 \\
\hline
\end{tabular}

Abbreviations: GA, general anesthesia; LA, local anesthesia; SA, spinal anesthesia.

Moreover, if the suture line is off midline, then the results are better with lower recurrence rate and wound dehiscence.

Recurrence of this disease is mainly due to incomplete elimination of the primary SPSD or due to persisting predisposing factors which include excessive hairiness, poor hygiene, and deep natal cleft. ${ }^{11-13,}$

We instilled hydrogen peroxide with methylene blue into the sinus opening to delineate the secondary and tertiary branches. The effervescence created by hydrogen peroxide facilitates the dye into the branches of the sinus, hence decreasing the chances of incomplete excision.

For reconstruction following excision, various flaps have been described. The z-plasty procedure was described by Monro and Macdermott. ${ }^{14}$ The disadvantage of this procedure was that part of the suture lining would lie in the midline which would predispose to recurrence. Another caveat of this procedure was a $20 \%$ incidence of flap tip necrosis. ${ }^{15}$

The w-plasty technique was described by Roth and Moorman in 1977. ${ }^{16}$ Again, part of the wound still remained in the midline and recurrence rate was as high as $8 \%$.

Off-midline closure was described by Karydakis. ${ }^{17,18}$ In his personal series of more than6,000 cases treated, he reported in 1992, the rate of recurrence as less than $2 \%$ and wound complications as $8 \%$. Similarly, Bascom has described "cleft lift" procedure to get off midline closure. ${ }^{19}$ Though the recurrence rate was low (1-4\%), complications like wound dehiscence were high (8.5-9\%). ${ }^{20}$

Similarly, flaps like the rhomboid and fasciocutaneous $\mathrm{v}$-y advancement flap have also reported a recurrence of $6 \%$ and $17 \%$, respectively. ${ }^{21-24}$

Thus, it is evident that several operative methods are available for the treatment of SPSD; however, no consensus exists on a gold standard method for treatment. ${ }^{9,25,26}$

The advantage of rotation flap over other methods, first, is that it has a simple design. It can be raised by any not-so-experienced surgeon. It is based on random pattern vascularity and, therefore, is reliable and heals well with almost no risk of failure.
Second, it gives wide exposure to the floor of the wound to visualize and excise any residual disease. Moreover, since large defects can be closed effectively, no compromise is needed on the excision.

Third, it flattens the natal cleft as it brings large amount of subcutaneous gluteal fat into the natal cleft region, thus greatly reducing a major risk factor in recurrence of SPSD.

Lastly, off-midline closure is achieved without tension on the suture line with aesthetically acceptable scar.

In our study, we have observed no recurrence in any of the patients. All our patients healed well except in two cases (3.85\%) that had minor wound complications and they also healed with conservative measures. The long term results are shown in (-Figs. 8 and 9).

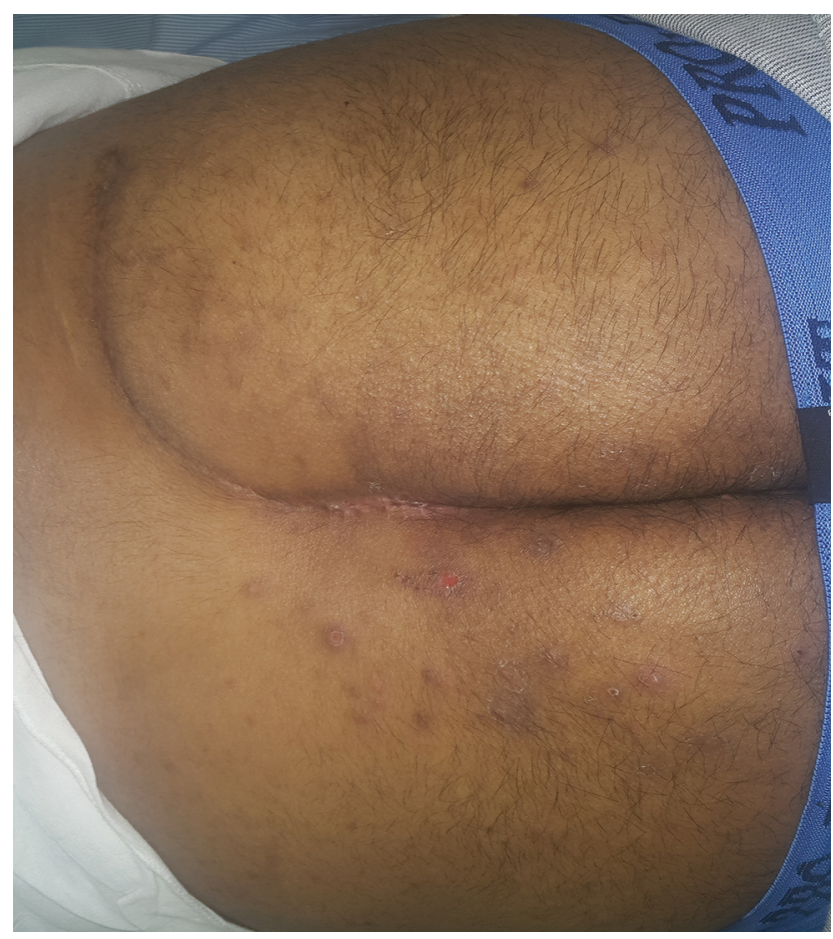

Fig. 8 Long-term results 2 . 


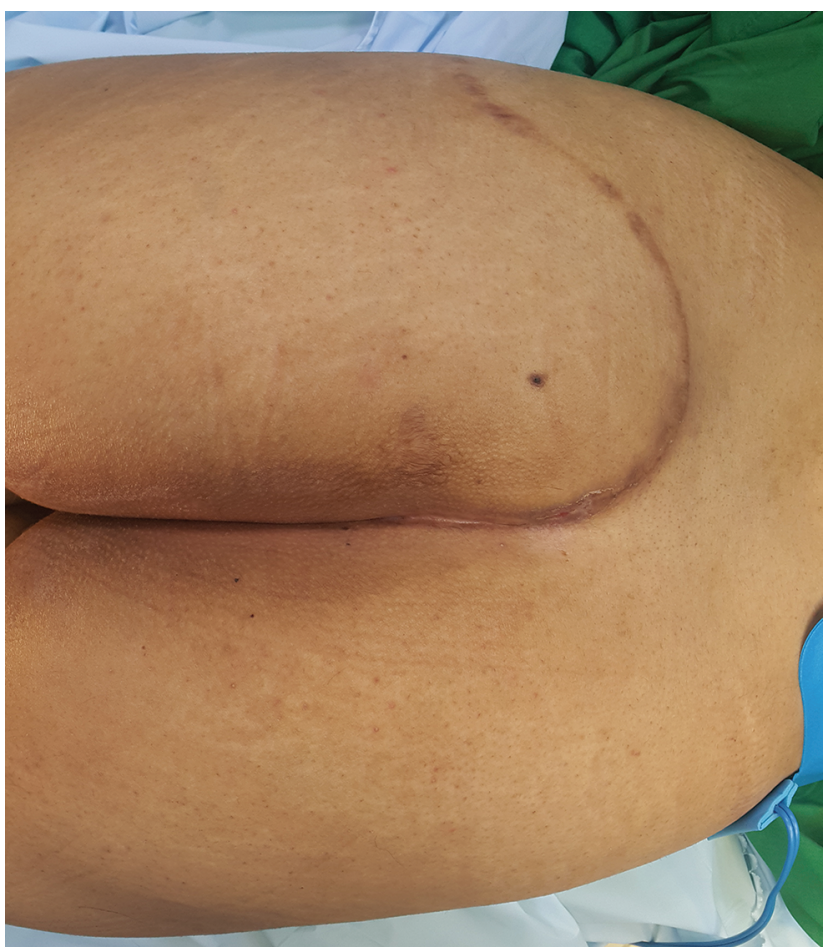

Fig. 9 Long-term results 3.

Table 1 represents the compilation of results of previous studies along with this study.

\section{Conclusion}

In our experience, single-stage surgical excision of the sinus tract using hydrogen peroxide and methylene blue for delineation and closure of the excision defect with rotation flap is a credible treatment option for SPSD.

\section{Financial Disclosure}

None.

\section{Conflict of Interest}

None declared.

\section{References}

1 Sondenaa K, Andersen E, Nesvik I, Soreide JA. Patient characteristics and symptoms in chronic pilonidal sinus disease. Int J Colorectal Dis 1995;10(1):39-42

2 Akinci OF, Bozer M, Uzunkoy A, Duzgun SA, Coskun A. Incidence and aetiological factors in pilonidal sinus among Turkish soldiers. Eur J Surg 1999;165(4):339-342

3 Akinci OF, Kurt M, Terzi A, Atak I, Subasi IE, Akbilgic O. Natal cleft deeper in patients with pilonidal sinus: implications for choice of surgical procedure. Dis Colon Rectum 2009;52(5):1000-1002

4 EsserJFS. Studies in plastic surgery of the face: I. Use of skin from the neck to replace face defects. II. Plastic operations about the mouth. III. The epidermic inlay. Ann Surg 1917;65(3):297-315

5 Lamke LO, Larsson J, Nylen B. Treatment of pilonidal sinus by radical excision and reconstruction by rotation flap surgery of Z-plasty technique. Scand J PlastReconstr Surg 1979;13(2):351-353

6 Omer Y, Hayrettin D, Murat C, Mustafa Y, Evren D. Comparison of modified Limberg flap and modified elliptical rotation flap for pilonidal sinus surgery: a retrospective cohort study. Int J Surg 2015;16(Pt A) :74-77

7 ImreJ. Lidplastik und Plastiche Operationen und anderer Weichteile des Gesichts. Budapest, Hungary: Studium, Verlag; 1928

8 Bascom J. Skin flaps for pilonidal disease. Ann PlastSurg 1998; 41(3):338

9 Johnson EK. Expert commentary on pilonidal disease: management and definitive treatment. Dis Colon Rectum 2018;61(7):777-779

10 Rao MM, Zawislak W, Kennedy R, Gilliland R. A prospective randomised study comparing two treatment modalities for chronic pilonidal sinus with a 5-year follow-up. Int J Colorectal Dis 2010;25(3):395-400

11 Abu Galala KH, Salam IM, Abu Samaan KR, et al. Treatment of pilonidal sinus by primary closure with a transposed rhomboid flap compared with deep suturing: a prospective randomised clinical trial. Eur J Surg 1999;165(5):468-472

12 Sevinc B, Karahan O, Okus A, Ay S, Aksoy N, Simsek G. Randomized prospective comparison of midline and off-midline closure techniques in pilonidal sinus surgery. Surgery 2016;159(3):749-754

13 Akca T, Colak T, Ustunsoy B, Kanik A, Aydin S. Randomized clinical trial comparing primary closure with the Limberg flap in the treatment of primary sacrococcygeal pilonidal disease. $\mathrm{Br}$ J Surg 2005;92(9):1081-1084

14 Monro RS, McDermott FT. The elimination of causal factors in pilonidal sinus treated by Z- Plasty. Br J Surg 1965;52:177-181

15 Toubanakis G. Treatment of pilonidal sinus disease with the Z-plasty procedure (modified) Am Surg 1986;52(11):611-612

16 Roth RF, Moorman WL. Treatment of pilonidal sinus and cyst by conservative excision and W-plasty closure. Plast ReconstrSurg 1977;60(3):412-415

17 Karydakis GE. Easy and successful treatment of pilonidal sinus after explanation of its causative process. Aust $\mathrm{N} \mathrm{Z}$ J Surg 1992;62(5):385-389

18 Karydakis GE. New approach to the problem of pilonidal sinus. Lancet 1973;2(7843):1414-1415

19 Bascom J, Bascom T. Failed pilonidal surgery: new paradigm and new operation leading to cures. Arch Surg 2002;137(10):1146-1150, discussion 1151

20 Kitchen PR. Pilonidal sinus: experience with the Karydakis flap. Br J Surg 1996;83(10):1452-1455

21 Khatri VP, Espinosa MH, Amin AK. Management of recurrent pilonidal sinus by simple V-Y fasciocutaneous flap. Dis Colon Rectum 1994;37(12):1232-1235

22 Berkem H, Topaloglu S, Ozel H, et al. V-Y advancement flap closures for complicated pilonidal sinus disease. Int J Colorectal Dis 2005;20(4):343-348

23 Milito G, Cortese F, Casciani CU. Rhomboid flap procedure for pilonidal sinus: results from 67 cases. Int J Colorectal Dis 1998;13(3):113-115

24 Harries RL, Alqallaf A, Torkington J, Harding KG. Management of sacrococcygeal pilonidal sinus disease. Int Wound J 2019;16(2):370-378

25 Stauffer VK, Luedi MM, Kauf P, et al. Common surgical procedures in pilonidal sinus disease: a meta-analysis, merged data analysis, and comprehensive study on recurrence. Sci Rep 2018;8(1):3058

26 Khanna A, Rombeau JL. Pilonidal disease. Clin Colon Rectal Surg 2011;24(1):46-53 